Onkologe 2021 27 (Suppl 1):S2-S4

https://doi.org/10.1007/s00761-021-01023-5

Angenommen: 28. Juli 2021

(c) Springer Medizin Verlag $\mathrm{GmbH}$, ein Teil von Springer Nature 2021

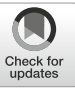

\section{Autoren}
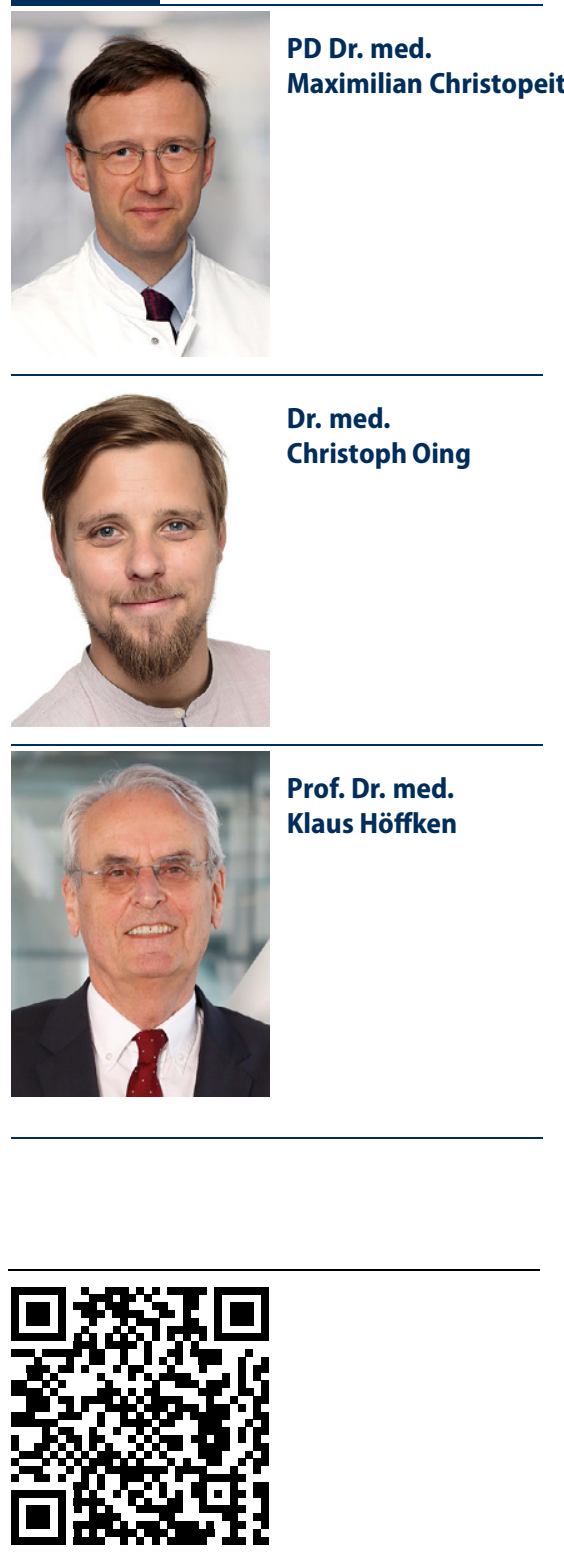

QR-Code scannen \&Beitrag online lesen

\title{
„Per aspera ad astra“ ... die Facharztreife
}

\author{
Maximilian Christopeit ${ }^{1} \cdot$ Christoph Oing ${ }^{2} \cdot$ Klaus Höffken $^{3}$ \\ ' Innere Medizin II | Hämatologie, Onkologie, Klinische Immunologie und Rheumatologie, \\ Universitätsklinikum Tübingen, Tübingen, Deutschland \\ ${ }^{2}$ Universitäres Cancer Center Hamburg (UCCH), II. Medizinische Klinik \& Poliklinik, Universitätsklinikum \\ Hamburg-Eppendorf, Hamburg, Deutschland \\ ${ }^{3}$ Berlin, Deutschland
}

Liebe Kolleginnen und Kollegen,

Sie halten eine auf Kasuistiken basierende Frage-Antwort-Sammlung zum Fachgebiet der Hämatologie und Medizinischen Onkologie in Ihren Händen, die der Vorbereitung auf die Facharztprüfung dienen soll und sich in eine Reihe ähnlicher Sonderausgaben entsprechender Periodika aus dem Springer-Verlag reiht.

Natürlich existieren zur Vorbereitung auf die "Reifeprüfung" einige ausgezeichnete Bücher und Kurse, ferner sind auch diverse Leitlinien sehr gut für das Facharzttraining geeignet.

Klinisches Handeln ist jedoch immer ein Arbeiten mit und am Menschen. Das Verständnis einer Erkrankung und ihrer Behandlung reift in der Auseinandersetzung mit dem „Verlauf am Beispiel“ am profundesten. Darum reicht die Beschäftigung mit einem einzelnen dieser Formate - Lehrbuch, Leitlinie, Kurs - ebenso wenig zur Vorbereitung auf die Prüfung und die eigenverantwortliche fachärztliche Führung der Hilfesuchenden wie das alleinige Studium der hier präsentierten Kasuistiken. Es liegt auf der Hand, dass eine Vorbereitung auf eine Facharztprüfung sich nicht in der Lektüre von Kasuistiken erschöpft. Unser unbedingter Rat ist, eine die gesamte Zeit der Facharztausbildung begleitende permanente Auseinandersetzung mit den Inhalten zu suchen und sich in der Zeit der Facharztausbildung auf die inhaltlichen Tätigkeiten zu fokussieren. Lapidar gesagt, die eigene Auswertung einer Knochenmarkaspiration ist noch wertvoller als die Perfektion der Technik der Knochenmarkpunktion selber. Die Binsenweisheit „Repe- titio est mater studiorum" gilt, und daher haben die Richtzahlen in den Facharztprüfungsordnungen sowie der Ausdruck „Facharztreife" ihre Berechtigung.

Aus unserem Fach „Innere Medizin und Hämatologie und Onkologie" wurden mit viel Forschungsfreude und Elan Paradigmen gesetzt; man denke nur an den $\mathrm{Ho}$ merun von der Beschreibung des Philadelphia-Chromosoms bis zur Heilung/ Chronifizierung der CML mit einer oralen Therapie. Heute sprechen wir von „tumoragnostischen Therapien", stehen gegebenenfalls vor einer erneuten Umwälzung des Fachs und sind voller Hoffnung, dass eine zunehmende Anzahl Neoplasien heilbar sein wird. Bei aller Forschungsaffinität ist unser Fach zuallererst ein klinisches Fach. Die Fähigkeit, den körperlichen und seelischen Nöten unserer Patienten und ihrer Angehörigen adäquat zu begegnen, speist sich unter anderem auch aus der Sicherheit im Fachwissen, weil es das Bewusstsein über Chancen, Risiken und Grenzen des Verlaufs einer Erkrankung trägt.

Ebenso bieten unsere Kasuistiken auch Kollegen nach der Facharztprüfung die Möglichkeit, diese zu nutzen und beständig weiter zu lernen. Die Fortsetzung der hier präsentierten ersten 23 Kasuistiken mit der Erweiterung um weitere Entitäten, Situationen und Fertigkeiten ist geplant.

Alle Lerninhalte finden Sie natürlich auch digital. Sie haben die Möglichkeit, diese als responsive E-Learning-Kurse angereichert mit kompaktem Hintergrundwissen unterhttp://www.springermedizin. de/facharzttraining z.B. am Smartphone oder Tablet durchzuarbeiten und dabei Ihren Lernfortschritt zu verfolgen. 
Hier steht eine Anzeige.

黑 Springer 


\section{Editorial}

Zusammenfassend hoffen wir, dass die Auswahl der Kasuistiken und die Beschreibung der dahinterstehenden Erkenntnisse Ihnen als ergänzendes Lehrmaterial in der Vorbereitung auf die Facharztprüfung und darüber hinaus dienen. Es muss nicht weiter betont werden, dass das Erlernen und Erinnern der kasuistikbezogenen Fragen keine Garantie für eine erfolgreiche Prüfung sein kann. Aber wir sind davon überzeugt, dass Kasuistiken den klinischen Alltag in all seiner Variabilität am besten wiedergeben und dem Format der Facharztprüfung Rechnung tragen. Wir wollen dieses Format weiter pflegen und sind für Anregungen, Kommentare und Beiträge dankbar.

Und nicht zuletzt wünschen wir, dass wir Sie auf der Grundlage des eingehenden Studiums (per aspera) dieses und weiterer Sonderhefte demnächst als Facharztkolleginnen und Facharztkollegen (ad astra) begrüßen können.

Maximilian Christopeit und Christoph Oing Herausgeber der Rubrik „Facharzttraining"

\section{Klaus Höffken}

Für die Herausgeber

\section{Korrespondenzadresse}

PD Dr. med. Maximilian Christopeit Innere Medizin II | Hämatologie, Onkologie, Klinische Immunologie und Rheumatologie, Universitätsklinikum Tübingen

Otfried-Müller-Straße 10, 72076 Tübingen,

Deutschland

maximilian.christopeit@med.uni-tuebingen.de

\section{Dr. med. Christoph Oing}

Universitäres Cancer Center Hamburg (UCCH), Il. Medizinische Klinik \& Poliklinik, Universitätsklinikum Hamburg-Eppendorf Martinistraße 52, 20246 Hamburg, Deutschland

\section{Prof. Dr. med. Klaus Höffken}

Welserstraße 21, 10777 Berlin, Deutschland

Interessenkonflikt. M. Christopeit, C. Oing und K. Höffken geben an, dass kein Interessenkonflikt besteht.

\section{Die ersten $\mathbf{1 2}$ Fälle mit Fokus Hämatologie}

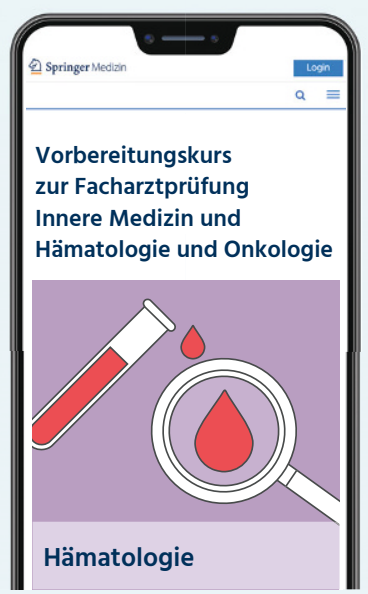

Starten Sie auch online oder in der App mit Ihrer Prüfungsvorbereitung!

Die ersten 12 Fälle aus dem breiten hämatologischen Tumorspektrum liegen für Ihr Training vor.

Der Zugang ist nach Registrierung bei SpringerMedizin.de kostenlos!

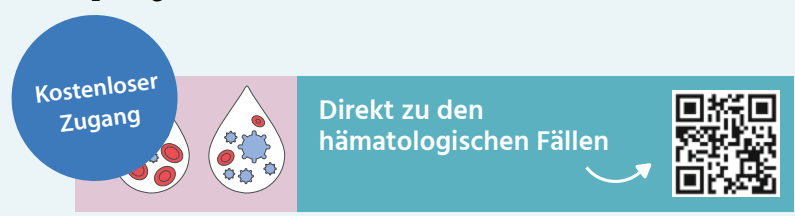

SpringerMedizin.de/Facharzttraining 